\title{
Ionization induced electron injection in laser wakefield acceleration
}

\author{
Min Chen ${ }^{1}$ and Zheng-Ming Sheng ${ }^{1,2}$ \\ ${ }^{1}$ Key Laboratory for Laser Plasmas (Ministry of Education) and Department of Physics, \\ Shanghai Jiao Tong University, Shanghai, 200240, China and \\ ${ }^{2}$ SUPA, Department of Physics, University of Strathclyde, Glasgow G4 ONG, UK
}

\begin{abstract}
In this chapter we describe and discuss a special technique in laser wakefield acceleration: electron injection by use of field ionization of atoms to high orders. Compared with other electron injection schemes in LWFA, this scheme shows the merits of relatively simple operation and controllable final beam quality. In the singlecolor laser ionization injection scheme, we show quasi-monoenergetic electron acceleration is possible through the control of laser self-focusing, which includes self-focusing accommodated ionization injection or ionization injection suppression, depending on the initial laser plasma parameters. In these processes the effective acceleration length can be controlled within a hundred micrometers range and the absolute energy spread of the beam can be controlled within tens of $\mathrm{MeV}$. In the two-color laser ionization injection scheme, we show the effective injection length can be furtherly reduced to tens of micrometers length, and the absolute energy spread of the electrons can be reduced to a few $\mathrm{MeV}$, i.e. the relative energy spread can be less than $0.5 \%$ for a beam with peak energy of $350 \mathrm{MeV}$. A further interesting result is the generation of multi-color electron bunches by use of two-color lasers. These electrons can be used for multi-color X-ray generation through laser beam Thomson scattering. It is also found that the transverse emittance of the electron beam can be significantly reduced by a different two-color ionization injection scheme, in which the wake excitation and electron injection are made by using lasers with different wavelength, respectively. The final transverse emittance of the beam can be less $10 \mathrm{~nm}$ rad. Some recent progresses wordwide in experimental demonstrations of ionization injection are reviewed briefly with special attention to the beam quality improvement. Some prospects of ionization injection for practical applications in laser wakefield accelerators are also discussed.
\end{abstract}

PACS numbers: 52.38.Kd, 52.65.Rr, 41.75.Jv, 52.38.-r

\section{PRINCIPLE OF IONIZATION INJECTION}

Laser or beam driven wakefield acceleration as demonstrated in world wide laboratories is a very powerful and promising new technology for advanced electron acceleration, radiation source, therapy, and colliders [1-4]. Currently, one of the most challenging issues to overcome the limits towards applications is to substantially improve the beam quality. For stable, high quality, high charge beam acceleration both wakefield and electron motion process should be well controlled. Wakefield control is related to the laser driver control, including guiding and mode shaping. As for the electrons, besides the beam dynamics during the acceleration, the injection stage is tremendously important, which directly affects the final beam charge, energy spread and emittance and is therefore of primary importance.

As we know, besides the high acceleration gradient, the wakefield also has a very high phase speed (closed to the speed of light) and small spatial scale (tens of micro meters). To inject an electron beam into such a high speed small structure and make it to be trapped are extremely challenging. Many kinds of injection schemes have been proposed to pursue controllable injection. By adding a plasma density downramp region along the driver propagation path, the phase speed of the wakefield can be slowed down and then some of the background thermal electrons maybe trapped by the slow wave and then be accelerated to high energy. The other way for electron injection is to boost the speed of some of the background electrons. If the pre-accelerated electrons are faster than the wave when they are located at the end of the wake bucket, the electrons can be trapped inside of the bucket and be continuously accelerated until they enter into the deceleration phase. To this purpose, a few pre-acceleration mechanisms have been already considered. Among them, colliding pulse injection is the most promising method and it has already been extensively theoretically studied and experimentally demonstrated in several labs around the world [5, 6].

Here, besides the above mentioned two injection methods, we will introduce a third, simple injection mechanism i.e. ionization injection. Before the detailed analysis of ionization injection, let us recall the injection theory for one dimensional wakefield which was firstly described by Esarey et al. in 1995 [7]. In one dimensional approximation the trapping of a test electron can be well described by the Hamiltonian $H(\gamma, \psi)=\gamma-\gamma \beta \beta_{p}-\phi(\psi)$ or

$$
H(u, \psi)=\left(\gamma_{\perp}^{2}+u^{2}\right)^{1 / 2}-\beta_{p} u-\phi(\psi)
$$

where $\beta$ is the longitudinal velocity of the particle normalized to c, $u=\gamma \beta$ is the normalized longitudinal momentum, $\beta_{p} \simeq$ $1-\omega_{p}^{2} / 2 \omega_{0}^{2}$ is the normalized phase velocity of the plasma wave, $\psi=k_{p} \xi=k_{p}\left(x-\beta_{p} t\right)$ is the plasma wave phase with $k_{p}=\sqrt{4 \pi e^{2} n_{0} / m_{e} c^{2}}$, and $\phi=e \Phi / m_{e} c^{2}$ is the electrostatic potential of the plasma wave normalized to the electronic rest energy, $\gamma_{\perp}=\sqrt{1+u_{\perp}^{2}}$ is the transverse Lorentz factor and $u_{\perp}$ is the normalized transverse momentum of the electron, $\gamma=\sqrt{\gamma_{\perp}^{2}+u^{2}}$ is the Lorentz factor of the electron. 
Usually each electron moves along a trajectory in the phase space with a constant H. Its momentum as a function of the Hamiltonian and the wake potential is

$$
u=\beta_{p} \gamma_{p}^{2}(H+\phi) \pm \gamma_{p}\left[\gamma_{p}^{2}(H+\phi)^{2}-\gamma_{\perp}^{2}\right]^{1 / 2}
$$

The particle may move in a closed (trapped) orbit or an open (untrapped) orbit depending on its initial position in the phase space. The separatrix orbit defining these two orbit families is given by $H\left(\gamma_{s}, \psi\right)=H\left(\gamma_{p}, \psi_{\min }\right)$, where $\phi\left(\psi_{\min }\right)=\phi_{\min }$, i.e.,

$$
H_{s}=\gamma_{\perp}\left(\psi_{\min }\right) / \gamma_{p}-\phi_{\min }
$$

If one puts $H_{s}$ from Eq. 3 into Eq. 2 , one can see $\beta\left(\psi_{\min }\right)=\beta_{p}$, which means that the electron in this obit has the same velocity as the wave at $\psi=\psi_{\text {min }}$. If its velocity at this point is a little bit larger than $\beta_{p}$, it moves into the acceleration phase and can get energy from the wake moving forward in the wave rest frame. It will be accelerated until it goes into the dephasing region. Otherwise it slips to the deceleration phase. This is just the meaning of the separatrix orbit.

Usually an electron will be in a trapped orbit provide $H \leq H_{s}$. For an electron initially at rest before the laser pulse, $H=1$. For an initially warm plasma, $H=H_{t} \simeq 1-\beta_{p} u_{t}$, where $u_{t} \ll 1$ is the initial non-relativistic thermal momentum. If the electrons transverse momentum is 0 , then trapping occurs for $\phi_{\min } \leq-1+\gamma_{p}^{-1}+\beta_{p} u_{t}$ which sets a minimum intensity threshold for the wake.

The wake potential is determined by the driver pulse and plasma parameters. In one dimensional geometry, the quasi-static laser driven plasma wave can be calculated according to the following equation:

$$
\frac{\partial^{2} \phi}{\partial \psi^{2}}=\gamma_{p}^{2}\left\{\beta_{p}\left[1-\frac{1+a^{2}}{\gamma_{p}^{2}(1+\phi)^{2}}\right]^{-1 / 2}-1\right\} .
$$

where $a=e A / m c^{2}$ is the normalized vector potential of the driver laser pulse and it can be calculated from the laser intensity (I) as $a^{2}=3.6 \times 10^{-19}(\lambda[\mu \mathrm{m}])^{2} I_{0}\left[\mathrm{~W} / \mathrm{cm}^{2}\right]$, assuming circular polarization, where $\lambda$ is the laser wavelength. A typical wakefield and its potential are shown in Fig. 1(a) by the red and blue lines, respectively.

Eq. 1 to Eq. 4 give a full description of the wake excitation and particle dynamics for one dimensional wakefield acceleration. Ionization injection in one dimensional case can also be described by this theory. The ideal of ionization injection is firstly mentioned by Umstadter et al. in 1997 at the end of his paper on ponderomotive force injection, where he said that "...Instead of using a fully ionized plasma (as above), one would use a medium with deeply bound inner shell electrons, which have an appearance intensity for tunneling ionization below that of the injection pulse but above that of the pump pulse ..." [8] The first detailed research work on ionization injection is done by Chen et al. in 2006 [9], where we used a double-pulse scheme. The first laser pulse works as a driver to excite a strong wakefield in a plasma, a second transversely propagated laser pulse ionizes the internal electrons of the high $\mathrm{Z}$ gas inside the wakefield at an appropriate time. In that paper, the contribution from ionization and ponderomotive force injections are compared in detail. Later on, the ionization injection scheme has been successfully generalized to a single laser pulse condition in which the wake excitation and ionization injection are performed by the same laser pulse. A lot of experimental studies have demonstrated this idea since 2010. Before that, ionization has already been used for electron injection in beam-driven plasma wakefield acceleration experiment.

Detailed 1D ionization injection theory for a single-pulse-driven injection scheme can be found in a paper written by Chen $e t$ $a l$. [10]. The only difference with that theory is the nonzero component of the particles' initial transverse momentum $u_{\perp}$ which is usually negligible in a normal plasma wave. For an electron ionized inside the laser pulse in the 1D geometry, the transverse canonical momentum is conserved $\partial_{\psi}=\left(u_{\perp}-a_{\perp}\right)=0$. So for an electron ionized at a wake phase $\psi_{i}, u_{\perp}(\psi)=a_{\perp}(\psi)-a_{\perp}\left(\psi_{i}\right)$, where we have assumed that the electron is born at rest, i.e. $u_{\perp}\left(\psi_{i}\right)=0$. Once it leaves the laser field $\gamma_{\perp}=\left(1+a_{\perp}^{2}\left(\psi_{i}\right)\right)^{1 / 2}$. So the Hamilton of the ionized electron is $H_{i}=\gamma_{\perp}\left(\psi_{i}\right)-\phi\left(\psi_{i}\right)=1-\phi\left(\psi_{i}\right)$. The trapping condition is

$$
-\left(\phi\left(\psi_{i}\right)-\phi_{\min }\right) \geq 1-\left(1+a_{\perp}^{2}\left(\psi_{\min }\right)\right)^{1 / 2} / \gamma_{p}
$$

which assumes that the laser pulse has zero intensity at the trapping position i.e., $a_{\perp}\left(\psi_{\min }\right)=0$. The left side of the in-equality represents the maximum energy that the electron can get from the wakefield after its ionization. It needs to be larger than a threshold value determined by the wake properties (see $\gamma_{p}$ ). Usually the wake itself determines $\phi_{\min }$ and $\gamma_{p}$. By using a mix of different gases, including high $\mathrm{Z}$ gas, the ionization position $\psi_{i}$ can be controlled. If ionization happens at a position with large wake potential, the trapping condition can be satisfied. Common gases such as Nitrogen, Oxygen, Argon can be used in mixtures for such kind of injection. A typical process is shown in Fig.1 in which the sixth and seventh electrons of Nitrogen are used for injection. The first five electrons are ionized in the early rising edge of the laser pulse where the wake potential is almost 0 . The last two electrons are ionized near the pulse peak at a position where the wake potential is high. The green line shows the distribution of $H_{s}-H$ for the electrons ionized at different positions. Electrons ionized where $H_{s}-H>0$ satisfy the injection condition. In this way only the two internal electrons of Nitrogen can be injected efficiently in the wake. Fig.1(b) shows a 2D-PIC simulation results of the injected electrons' trajectories related to the wake at two different time steps. As one 
(a)

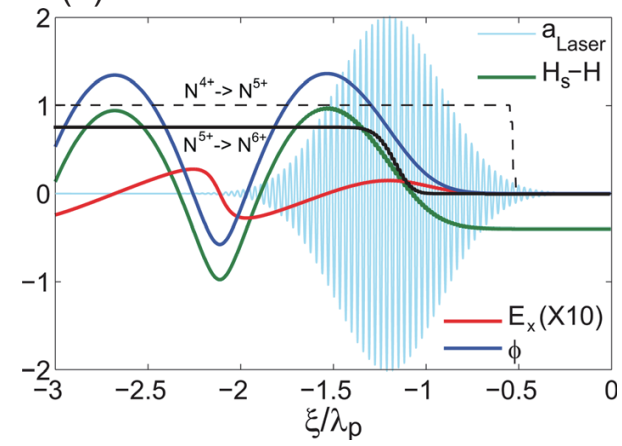

(b)

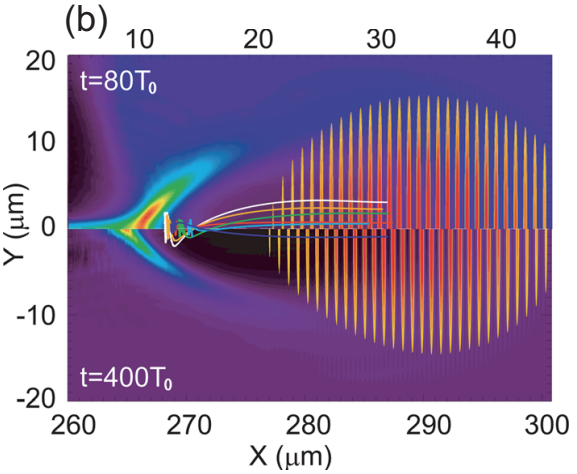

FIG. 1: Schematic view of ionization induced injection. (a)Analytical results for 1D ionization injection. Normalized laser vector potential (light blue/gray curve), wake potential (blue/up dark gray curve), wake electric field (red/lower gray curve), $H_{s}-H$ (green/middle gray curve), and degree of ionization versus $\xi / \lambda_{p}$. The dashed black curve shows the degree of ionization for $N^{4+} \rightarrow N^{5+}$, and the solid black curve shows $N^{5+} \rightarrow N^{6+}$. (b)2D-PIC simulation results of ionization induced injection. Typical trajectories of trapped electrons via ionization. Laser-plasma parameters are $a_{0}=2.0 ; L_{F W H M}=14.89 T_{0}$, focus spot size $W_{F W H M}=17.66 \lambda_{0}$, uniform plasma density with $n_{e}=0.001 n_{c}$, and mixed gas length $l=20 \lambda_{0}$. The Nitrogen concentration is $1 \%$. Top half shows $t=80 T_{0}$ and bottom half shows $t=400 T_{0}$.

can see the injected electrons to be injected are ionized closed to the peak of the laser pulse where the wake has a suitably high potential. Actually some of the electrons are ionized off-axis. This gives another source of transverse emittance in addition to the $u_{\perp}$ from ionization process.

Compared with a normal injection theory, the trapping condition is modified by including the effect of the residual transverse momentum resulting from the ionization process (see $a_{\perp}\left(\psi_{\min }\right)$ ). As we will show later it is just because of this residual momentum that electrons injected by ionization may have large transverse emittance. By using two-color laser pulses to separate wake excitation and injection processes, one can overcome this problems. Since the whole process involves many nonlinear phenomenons, such as laser propagation, wake excitation, ionization, electron trapping, and so on, a simple analytical theory cannot give correct and detailed information. Computational simulations and experiments are the main tools to study these processes. In the following we will discuss a variety of ionization injection schemes to obtain high quality (low energy spread, low transverse emittance) electron beams. Finally, we will show some recent experimental and theoretical progresses on this topic.

\section{USING IONIZATION INJECTION TO GET LOW ENERGY SPREAD ELECTRON BEAMS}

From above analysis, one can see that ionization injection is a relatively easy scheme to inject electrons in a wakefield. No external laser pulse or gas jet need to be installed. Just by mixing some high $\mathrm{Z}$ gas in He gas may lead to ionization injection once the laser and plasma have appropriate parameters. A few earlier experiments have already demonstrated this scheme. However, the final accelerated electron beam quality is not as good as other injection schemes [6]. The spectrum always shows a tail in the low energy part. The reason is that usually the laser path $L_{\text {injection }}$ into the mixed gas is larger than 1-mm. For electrons injected in wakefield, the distances between the initial injection positions can result in a big final energy spread $\delta \varepsilon$, with a maximum energy difference of $\delta \varepsilon^{M}=E_{x} \times L_{\text {in jection. }}$. Usually the wakefield amplitude $E_{x}$ is $\sim G e V / \mathrm{cm}$, so 1-mm separation in the injection distance leads to $100 \mathrm{MeV}$ energy difference. To reduce the absolute energy spread, effective injection length should be controlled and kept as short as possible. In this section two schemes are aimed to decrease the effective injection length. The first one is by using the self-evolution of the wakefield due to the unmatched laser pulse: the injection is switched off by varying the wake parameters out from the injection condition range. The second is by controlling the ionization itself through laser electric field intensity modulation. Both of them can lead to mono-energetic electron spectrum. The second way is able to reduce the energy spread down to less than $1 \%$ and can also provide multi-color electron bunches.

\section{A. Self-truncated ionization injection}

In the first section, we introduced the ionization injection theory in $1 \mathrm{D}$ geometry. In that condition, laser has no transverse evolution. However, in reality, both laser and wake experience transverse evolutions, such as laser pulse self-focusing and defocusing, accompanied by wake elongation and expansion. These phenomena have already been used for controlling selfinjection in LWFA [11]. Recently this mechanism has been used in ionization injection. Zeng et al. studied the injection process with an unmatched laser [12]. There we found a simple method to cut the ionization injection length down to a few hundred micrometers in single stage with a single pulse. Finally we are able to produce an electron beam with $25.5 \mathrm{pC}$ in charge and $8 \%$ 

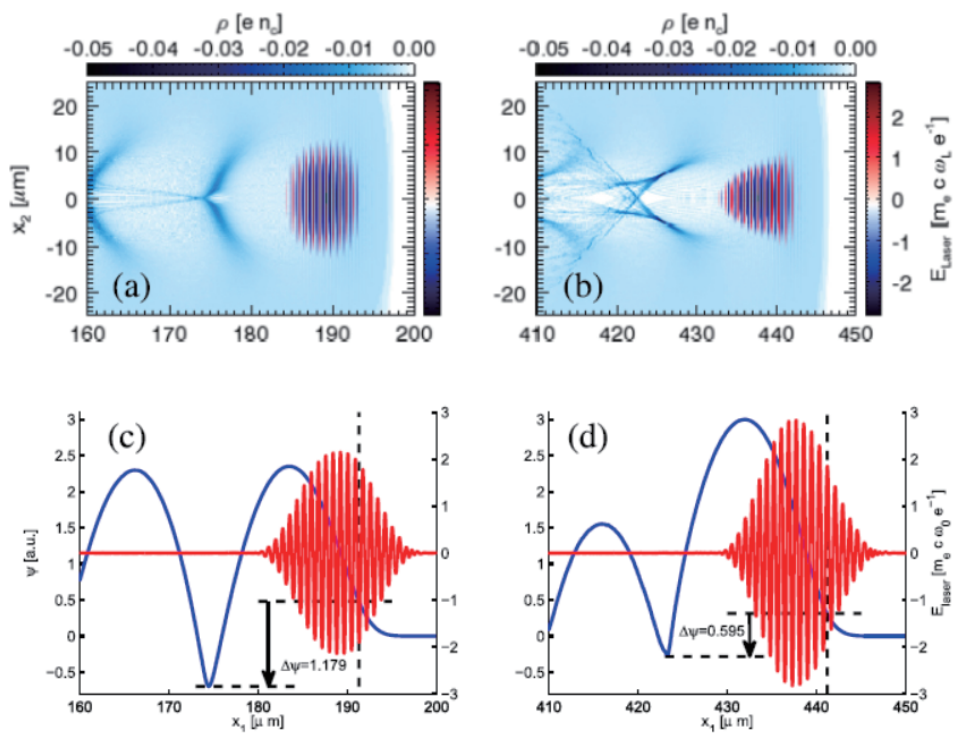

FIG. 2: (a) and (b) Electron density and laser electric field distribution at the laser propagation distance of 200 and $450 \mu m$, respectively. (c) and (d) Laser (red lines) and pseudo-potential (blue lines) line-outs at these two time steps. Densities are normalized by $n_{c}$ and electric fields are normalized by $E_{0}=m_{e} c \omega_{L} e^{-1}=10^{12} \mathrm{~V} / \mathrm{m}$. The dashed lines show the ionization starting point of nitrogen inner shell and the fall to the bottom of the potential well $(\Delta \psi=1.179$ and 0.595 , respectively).
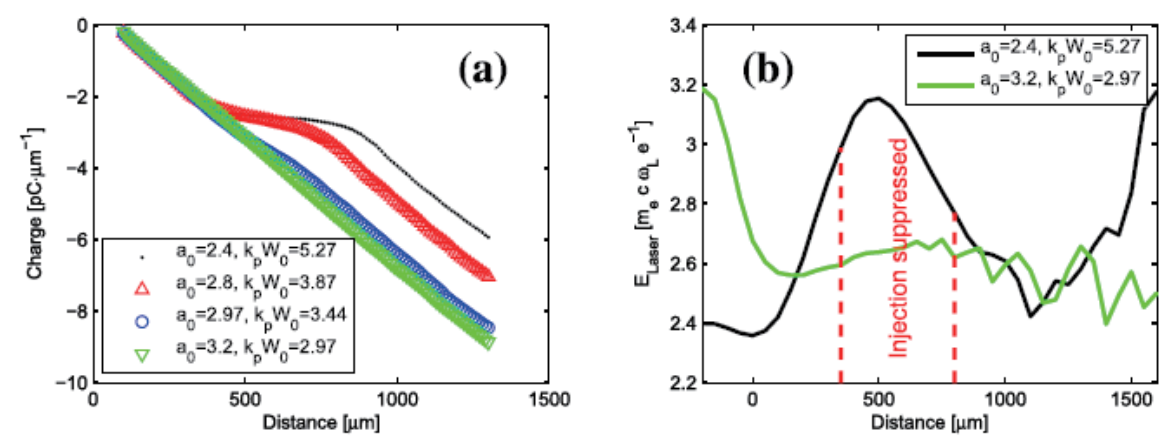

FIG. 3: (a) Injected charge number vs. laser propagation distance from 2D PIC simulations with different normalized laser vector potential $a_{0}$ and normalized waist $k_{p} W_{0}$. The laser energies are kept the same in 2D slab geometry by keeping $a_{0}^{2} W_{0}$ a constant. (b) Laser peak amplitude evolution of two cases: black line for $a_{0}=2.4, k_{p} W_{0}=5.27$ and green line for $a_{0}=3.2, k_{p} W_{0}=2.97$. The red dashed lines indicate the region where the injection is suppressed for the black line case.

FWHM energy spread. To illustrate this mechanism a series of $2 \mathrm{D}$ simulation results are performed, a typical result is shown in Fig. 2. In the simulations a 800nm laser pulse with vector potential of $a_{0}=2.0$, waist of $k_{p} W_{0}=7.594$, pulse duration of $L_{F W H M}=33 f s$ are used. The background plasma density is $1.6 \times 10^{-3} n_{c}$ and mixed nitrogen atom density is relatively small and varies from $5 \times 10^{-6}$ to $1 \times 10^{-4}$. Snap shots of wakefield and laser pulse at two different acceleration distances are shown. At about $x=190 \mu m$, the ionization injection condition is satisfied with $\Delta \phi=1.179>1$. However at the distance $x \approx 440 \mu m$ the laser pulse is self-focused and the corresponding wake evolves significantly which makes $\Delta \phi=0.595<1$ and the injection condition has been broken. Thus the injection is self-truncated by the laser and wakefield evolutions. In this way the effective injection length is not determined by the mixed gas length (usually in the scale of $\mathrm{mm}$ ) but it is determined by the laser evolution time-scale which is in this case around $200 \mu \mathrm{m}$.

Obviously this kind of ionization injection depends on the evolution of the laser pulse. Experimentally one can vary the laser focusing spot size to control this process. Figure 3(a) shows such effects. In the 2D simulation, the laser energies are kept to be a constant by keeping $a_{0}^{2} W_{0}$ unchanged. Different spot size and corresponding laser intensity are used for testing runs. As one can see in some case (the green line) the laser evolution is very mild (see Fig. 3(b)) and electrons are continuously injected into the wake (see Fig. 3(a)). In this case the final electron spectrum is a plateau structure with hundred percent of energy spread. On the contrary, if the laser is strongly evolved like the black line case in Fig. 3. The injection happens at the beginning, then it is 


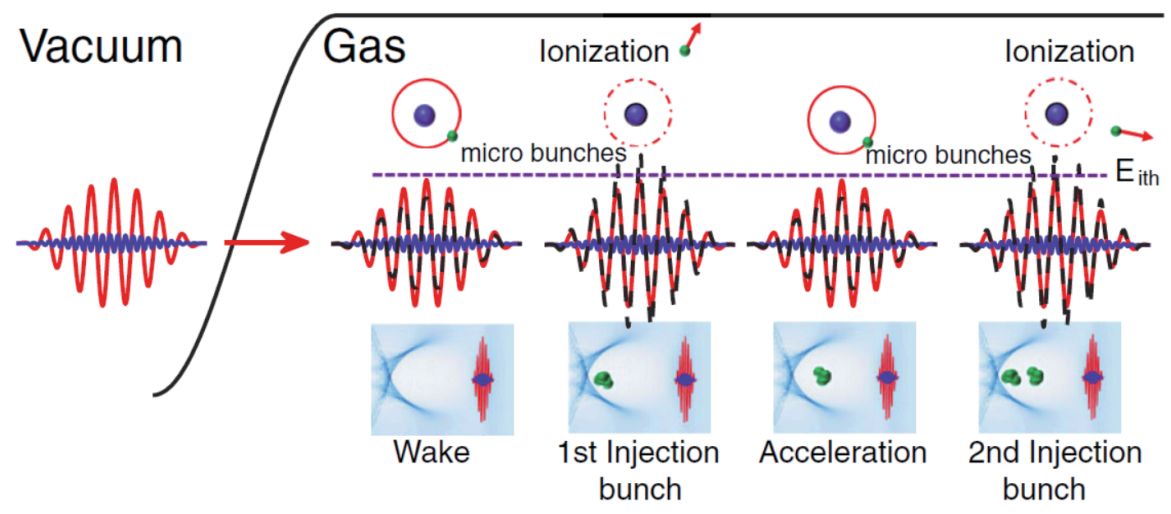

FIG. 4: Schematic view of dual color lasers trigged periodic injection in LWFA. A laser with base frequency $\omega_{1}$ (the red curves) and its harmonic $\omega_{2}$ (the blue curves) propagate in a mixed gas plasma. The dashed black curves show the superposition of the two frequency laser fields at different propagation distances. Laser parameters are chosen so that ionization-induced injection can be switched on when the beating is constructive and be switched off when the beating is destructive. In the plot, $E_{i t h}$ represents the effective threshold field for the high-Z gas inner shell ionization.

self-truncated and finally a second injection happens. The final spectrum shows two peaks, each one with narrow energy spread. Combining this technique with shortening (by mechanical fabrication) of the laser path in the gas-mix, electron spectrum with a single quasi-monoenergetic peak is possible. 3D-PIC simulation done by Zeng et al. proved how effective such a scheme can be [12]. A recent experiment done by Li et al. demonstrate this scheme. Monoenergetic electron beam with energy spread of $10 \%$ and central energy of $400 \mathrm{MeV}$ are reported [13].

\section{B. Two-color ionization injection}

The above self-truncated injection scheme provides a simple way for mono-energetic electron beam acceleration. However, since basically it relies on laser self-focusing it is a not well controllable method to get stable acceleration. Further, the energy spread is still too large for many applications. Besides controlling the wake properties, the other way is to control the ionization process. Xia et al. have used this to control ionization injection [14]. However in their case, the wake evolution effect is small. The laser intensity is tuned around the ionization threshold. Only when the self-focusing happens internal electrons of the injection gas are ionized and injected. Such process can also be used for mono-energetic beam acceleration. However, it is more difficult to tune the laser energy and the energy spread is still relatively large.

A two-color ionization injection scheme is recently been proposed by our group [15]. The idea is schematically shown in Fig. 4. Two laser pulses with vector potential of $a_{i}(z, t)=a_{i 0} \sin \left(\omega_{i} t-k_{i} z+\phi_{i}\right),(i=1,2)$ are used to excite the wake and trigger ionization injection, respectively. The injection pulse is a frequency-tripled laser. As two color pulses copropagate in the background plasma, the peak amplitude of the combined laser field is modulated in time and space during the laser propagation due to the plasma dispersion $\left(\omega_{i}^{2}=\omega_{p}^{2}+c^{2} k_{i}^{2}\right)$. Ionization injection only occurs when the peak amplitude exceeds a certain threshold. The threshold is exceeded for very short duration periodically at different propagation distances, leading to multiple ionization injections and separated electron bunches.

The combined laser electric field of the two pulses can be rewritten as

$$
E(\xi, s)=a_{10} \cos \left(\xi+\omega_{p}^{2} s / 2+\phi_{1}\right)+a_{20} \omega_{2} \cos \left(\omega_{2} \xi+\omega_{p}^{2} s / 2 \omega_{2}+\phi_{2}\right)
$$

where $\xi=\omega_{1}(t-z / c)$ and $s=\omega_{1} z / c$. One can see that the electric field is a periodic function of $s$ and $\xi$ and the period of $\mathrm{s}$ is $\Delta s=4 \pi / \omega_{p}^{2}\left(\omega_{2}-\omega_{2}^{-1}\right)$. Similarly to the peak amplitude evolution of the two color pulse, the ionization injection region can be broken into small pieces. By appropriately selecting the amplitude and the frequency of the two pulses, ionization injections can be limited to a few small separated regions. Zeng et al. has shown typical simulations for this scheme in their papers published in Physical Review Letters [15]. In the scheme they used Nitrogen as injection gas and laser pulses with $800 \mathrm{~nm}$ and $266 \mathrm{~nm}$ wavelengths, respectively.

A typical 2D simulation is shown in Fig 5, where laser parameters are: $a_{10}=1.46, a_{20}=0.162, L_{F W H M}=33 \mathrm{fs}$, $\mathrm{W}_{0}=$ $80 \mu \mathrm{m}, \mathrm{n}_{\mathrm{e}}=1.6 \times 10^{-3} \mathrm{n}_{\mathrm{c}}, \mathrm{n}_{\mathrm{N}}=1.6 \times 10^{-7} \mathrm{n}_{\mathrm{c}}$. The injection stage length (the length of mixed nitrogen) is $L_{i n j}=1 \mathrm{~mm}$ which is smaller than the laser self-evolution scale length. So the laser self-focusing and wake evolution effects can be negligible. A typical distribution of the injected bunches is shown in Fig. 5(a). The central positions of these bunches are spatially separated in this snapshot on a micrometer scale. The phase space distribution of the bunches are shown in Fig. 5(b), from which we see 

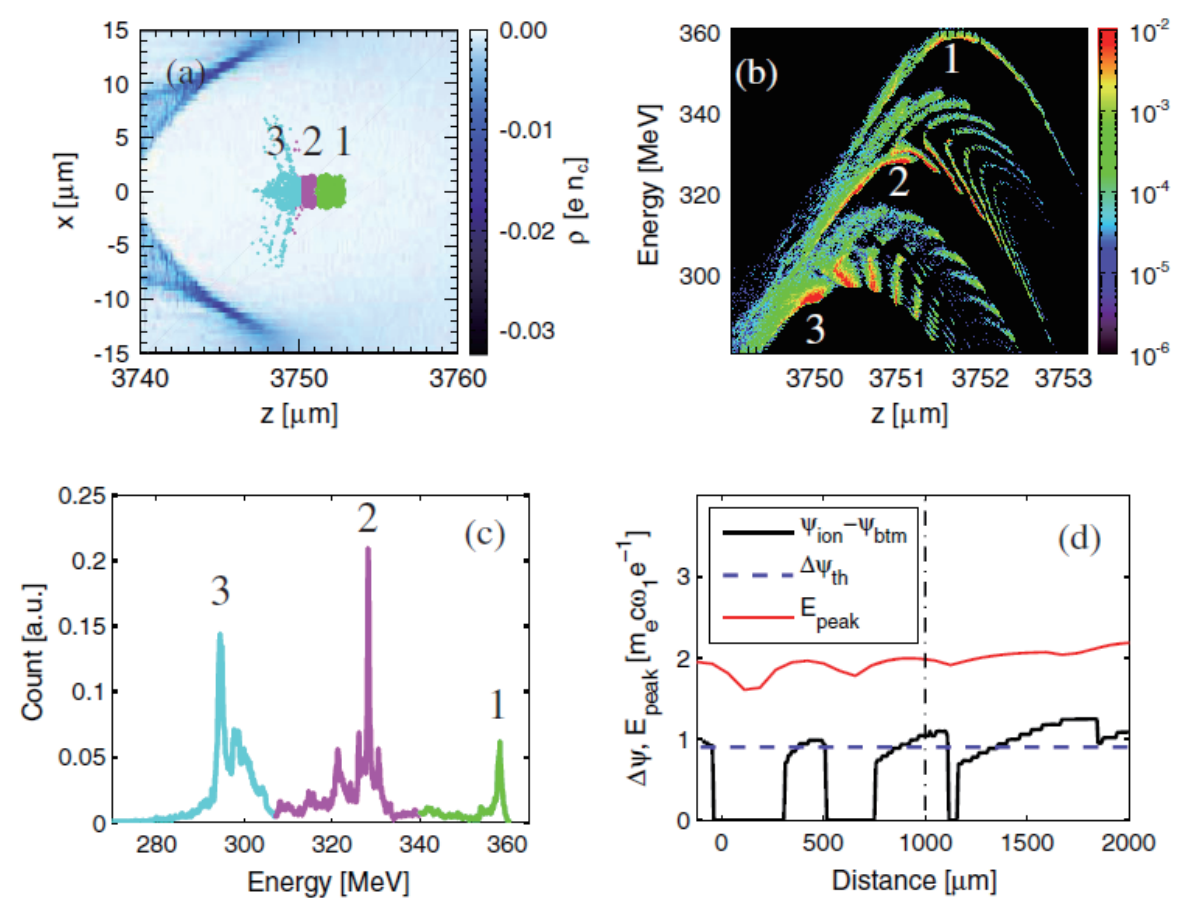

FIG. 5: 2D PIC simulations of the two-color ionization injection injection scheme. (a) The density snapshot at $z=3780 \mu m$. The colored dots show the locations of three electron bunches. (b) The energy and space distribution of the energetic electrons. (c) The spectrum of the injected electrons, showing three monoenergetic peaks. (d) The pseudopotential $(\psi)$ difference of the wake and the laser peak field evolution. The dash-dotted line is the separation from the mixed gas region to the pure helium region.

that within the second and the third bunches there are a few micro bunches which come from the overlapping of several peaks when the combined electric field is larger than the ionization threshold as schematically shown in Fig. 4. These bunches degrade the monochromaticity of the final beams, showing the pedestals between the peaks of the energy spectrum in Fig. 5(c). From the simulations they found that those pedestals can be reduced by using a shorter triple frequency laser pulse, which makes the inner shell ionization occurring only in a single peak. A simulation with a $10 \mathrm{fs}, 3 \omega$ laser gives a single injected electron bunch with final energy spread less than 0.2\% FWHM. In the simulation of Fig. 5, the whole spectrum is composed of three main peaks with equal separation of $30 \mathrm{MeV}$. The ionization injection analysis is shown in Fig. 5(d). The mixed gas locates on the left side of the dashed line. The intensity of the combined laser electric field is shown by the red curve. The pseudopotential differences and the threshold for ionization injection are shown by the black solid and blue dashed lines, respectively. The former is automatically set to be zero if ionization cannot occur. As one can see, ionization injection only happens in three regions, namely around the beginning, at about $500 \mu \mathrm{m}$, and $1000 \mu \mathrm{m}$. Each one occurs in a very limited region (tens of micrometers). This is consistent with the three injected electron bunches labeled by 1,2,3 in Fig. 5(a,b,c). More 3D simulations also demonstrate the effectiveness of such scheme. More detailed information can be found in Ref. [15].

Besides the extreme low energy spread of the electrons injected and accelerated by the above two-color ionization injection method, the scheme is also unique for generating multichromatic narrow energy-spread electron bunches which can be used for multi color x-ray generation through Thomson Scattering scheme. These are interesting for medical or material imaging applications. The multichromatic beams may also be interesting for radiotherapy. From comparing the characteristics of radio physics of the multi-fields full body irradiation by mixed-energy electron beam with single-energy electron beam, results show that multi-chromatic energy electron beams can not only meet the clinical treatment requirements for treating mycosis fungoides, but also shorten the treatment time compared to the single-energy electron beam radiation treatment [16]. The current challenge is to reduce the electron energy from $100 \mathrm{MeV}$ to a few MeV level [17].

\section{USING IONIZATION INJECTION TO GET LOW TRANSVERSE EMITTANCE ELECTRON BEAMS}

As we mentioned before, electrons ionized inside the laser pulse may carry a residual transverse momentum from ionization. These momenta increase the final beam transverse emittance $\left(\varepsilon_{N} \sim \gamma r_{b} p_{\perp} / p_{\|}=r_{b} \hat{p}_{\perp}\right)$, where $r_{b}$ represents the transverse beam size. Since the residual momentum $\hat{p}_{\perp} \propto a$, reducing the vector potential of the ionizing laser pulse or changing its direction 

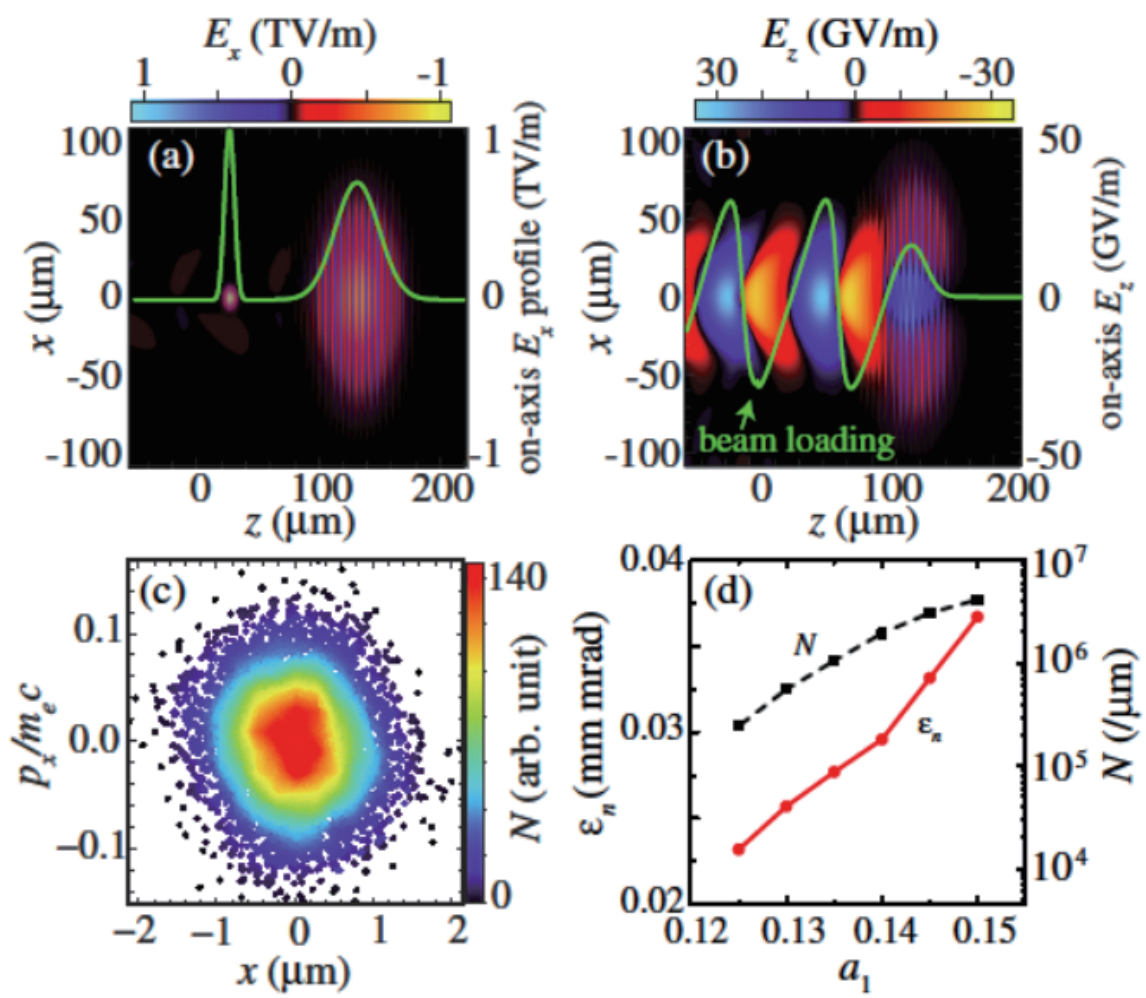

FIG. 6: (a) The electric field of the laser pulses (green curve is on-axis laser field profile), (b) the longitudinal wakefield (green curve is on-axis wakefield), and (c) the transverse phase space of the injected electrons, after the (mixed gas) ionization injection region. (d) The normalized transverse emittance $\varepsilon_{N}$ (red solid curve) and trapped electron number N (black dashed curve) versus the injection pulse amplitude $a_{1}$. See text for laser-plasma parameters.

are possible methods to lower the final beam emittance. In the following two corresponding schemes are introduced to get low transverse emittance within an ionization injection configuration.

\section{A. Two-color ionization injection}

From Eq. 4 one see that a driver pulse with larger vector potential $(a)$ can excite a stronger wakefield. This looks in contradiction with the low transverse emittance requirement (see before $u_{\perp}(\psi)=a_{\perp}(\psi)-a_{\perp}\left(\psi_{i}\right)$ ). However, if one uses two laser pulses to separate wake excitation from ionization injection, the contradiction no longer exists. Such scheme has recently been studied by $\mathrm{Yu}$ et al. [18] and $\mathrm{Xu}$ et al. [19]. In their scheme a low frequency (large wavelength, such as $\lambda_{0}=5 \mu \mathrm{m}$ or $C O_{2}$ laser $\left.\lambda_{0}=10.6 \mu \mathrm{m}\right)$ laser pulse, is used for wake excitation. Since the laser vector potential is $a=\sqrt{I\left(\mathrm{w} / \mathrm{cm}^{2}\right) / 1.38 \times 10^{18}} \lambda(\mu \mathrm{m})$, for a fixed laser pulse intensity, the longer the wavelength the larger the vector potential, which is better for wave excitation. On the contrary, a high frequency laser pulse is used to trigger ionization injection. This is because from $E \propto \partial A / \partial t$ one gets $a \propto E / \lambda$ and for similar ionization degree (similar electric field intensity $E$ ) the larger the wavelength the smaller the vector potential, which is better for keeping a small residual transverse momentum.

A typical two dimensional simulation of this scheme by Yu et al. is shown in Fig. 6. In the simulation Kr is used as injection gas.The electron density (after ionization by the pump laser) is fixed to $n_{0}=n_{e}+8 n_{K r}=2 \times 10^{17} \mathrm{~cm}^{-3}$, where $n_{e}$ is the electron density produced by ionization of the low-Z background gas (e.g. He gas), and the pump laser ionizes the $\mathrm{Kr}$ gas to $K r^{8+}$. The $\mathrm{Kr}$ gas locates from $z=-100 \mu \mathrm{m}$ to $z=0 \mu \mathrm{m}$, with a plateau of $50 \mu \mathrm{m}$ bordered by two ramp-like density profiles. The pump and injection pulses have the normalized vector potential like $a(\xi)_{0,1}=a_{0,1} \exp \left[-\left(\xi-\xi_{0,1}\right)^{2} / L_{0,1}^{2}\right]$, with amplitudes $a_{0}=1.17$ and $a_{1}=0.135$, wavelenghts $\lambda_{0}=5 \mu \mathrm{m}$ and $\lambda_{1}=0.4 \mu \mathrm{m}$, durations (FWHM) $T_{0}=92 f s$ and $T_{1}=16 f s$, and spot sizes $w_{0}=36 \mu \mathrm{m}$ and $w_{1}=5 \mu \mathrm{m}$, respectively. Different from the two-color scheme we discussed above to get low energy spread electron beam, here the two pulses are not overlapped with each other but they are separated by an optimal delay corresponding to $\left|\xi_{0}-\xi_{1}\right|=106.25 \mu \mathrm{m}$. So the electrons ionized by the second pulse are located in the trapped orbit of the wake excited by the first pulse.

Simulation shows that all the ionized electrons (with charge of $1.05 \times 10^{6} / \mu m$ ) are trapped in the wake. So they are lon- 

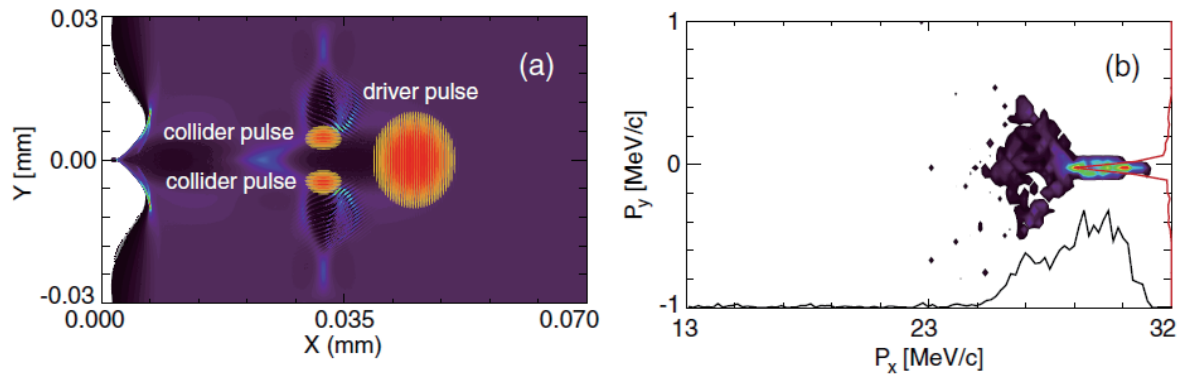

FIG. 7: (a) Plasma density and laser intensity distribution at $t=200 \mathrm{fs}$. (b) Phase space distribution $\left(P_{x}-P_{y}\right)$ of trapped preionized and laserionized electrons after the drive pulse propagated $0.79 \mathrm{~mm}$. The right red curve shows the transverse momentum spread after longitudinal integration. The lower black curve shows the beam energy spread.

gitudinally accelerated by the wake (see Fig. 6(a,b)). The transverse phase-space distribution of these electrons are shown in Fig. 6(c). As we can see most of the electrons have zero residual transverse momentum. This is because they are usually ionized at the peak of the injection laser electric field, where the laser vector potential is zero for a normal single color Gaussian pulse. Electrons ionized just off-peak of the laser electric field are trapped and produce the finite transverse momentum. The maximum momentum in Fig. 2(c) is $p_{x} / m_{e} c \simeq 0.17$, which is larger than the vector potential amplitude $a_{1}$ due to the effects of the transverse force of the wakefield and transverse ponderomotive force of the injection laser pulse. The rms beam radius and transverse momentum are $\sigma_{x} \simeq 0.55 \mu \mathrm{m}$ and $\sigma_{p_{x}} / m_{e} c=0.05$, respectively. Here $\sigma_{p_{x}}$ is an order of magnitude smaller than that observed in simulations of single-pulse ionization injection. The final transverse emittance of the electrons is $\varepsilon_{n}=0.028 \mathrm{mmmrad}$.

Similar idea was also studied recently by Xu et al. [19]. Instead of $5 \mu \mathrm{m}$ driver pulse, they used $\mathrm{CO}_{2}$ laser as a driver pulse. However, the basic idea is exactly the same. Detailed information can be found in Ref. [19].

\section{B. Ionization injection assisted by transverse colliding pulses}

As we see before the ionization induced transverse emittance is due to the transverse residual from the injection pulse generated during ionization. One may think that if this momentum is not fully transverse the emittance may be reduced. A recent modified ionization injection scheme proposed by Chen et al. just uses this idea [20,21]. The idea is schematically shown in Fig. 7(a). A driver pulse first excite a wave in the background plasma, then two identical frequency transverse laser pulses with polarization parallel to the driver pulse propagation direction are oppositely injected and overlapped at an appropriate phase of the wake. The combined laser field of the two transverse pulses is high enough to ionize the internal electrons of the injection gas (e.g. Nitrogen). Since the vector potential of the two pulses are along the longitudinal direction of the wake, the residual momentum is along this direction and no transverse momentum is introduced.

Chen et al. have studied this scheme by using 2D particle-in-cell simulations. A driver pulse with length of $L_{0}=6.0 T_{0}$, transverse size of $W_{0}=10 \lambda_{0}$ and intensity of $a_{0}=1.5$ is used. The injection pulses have temporal duration of $L_{1}=L_{2}=3.0 T_{0}$ and transverse sizes of $W_{1}=W_{2}=3.0 \lambda_{0}$. They have the same wavelength as the driver pulse $\left(\lambda_{0}=0.8 \mu \mathrm{m}\right.$ and intensities $a_{1}=a_{2}=0.8$. They collide with each other at the axis of the driver pulse propagation and at an optimal delay with the driver pulse which makes the electron injection possible. The background plasma has a density of $n_{\text {pre }-e}=0.001 n_{c}$. The density of the injection gas (Nitrogen) is $n_{N}=2.0 \times 10^{-6} n_{c}$. Simulation results shown $6.38 \times 10^{6} / \mu m$ electrons have been injected into the second wave bucket. The final beam transverse full-with-at-half-maximum (FWHM) momentum spread is $0.1 m_{e} c$ as shown in Fig. 7(b). The beam is quasi-monoenergetic with the peak energy of $29.32 \mathrm{MeV}$ and FWHM energy spread $\delta E \simeq 3.84 M e V$. The transverse momentum spread is an order smaller than the one by using an usual single pulse ionization injection scheme. Further optimal simulations show the minimum transverse emittance of the electron beam injected by this method can be as small as $0.08 \mathrm{mmmrad}$ which is far less than the normal value of 1 mmmrad.

It deserves to point out that, besides ionization induced electron injection into the wakefield, the transverse ponderomotive force of the two pulses may also lead to ponderomotive injection which is similar as the scheme proposed by Umstadter in 1996 [8]. To increase ionization injection percentage one needs to use two transverse laser pulses with identical frequency, otherwise transverse beat wave will be generated which accelerates electrons transversely, resulting in a high transverse momentum of the electrons. Two pulses with different frequency may be used to increase the transverse momentum of the injected electron beam to make stronger betatron radiation.

This idea was later introduced to beam driven wakefield acceleration by Li et al. [22]. Since in a beam driven case, the field of the beam is much smaller than the driver laser's electric field, gases with very low ionization potential can also be used as injection gas such as Helium. The beam ionizes only the electrons with lower ionization potential. Two transverse pulses, with even smaller amplitudes than in the case above, can be used to ionize the internal electrons of He and induce ionization 


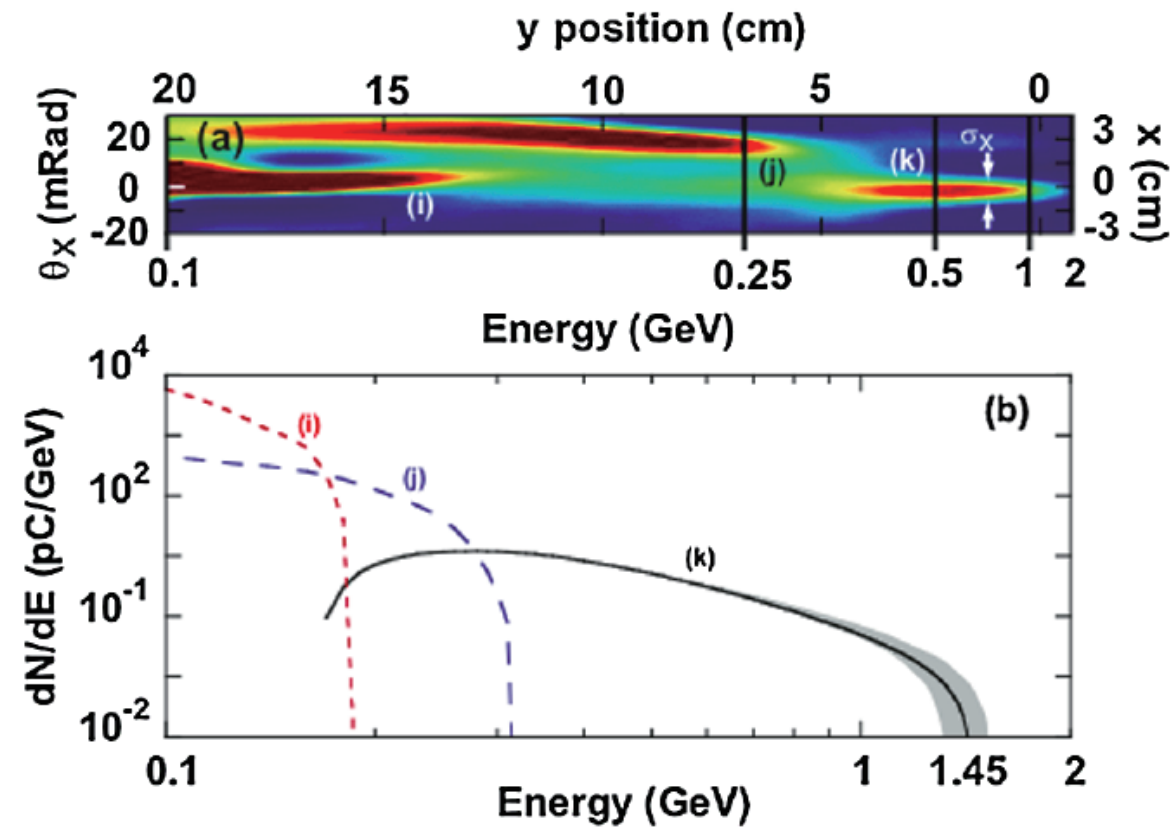

FIG. 8: (a)Raw electron data from for Clayton et al.'s experiments [26]. (b) Energy spectrum for the three features in the energy measurement.

injection. This makes the final transverse electron beam emittance even smaller. In their studies ultrashort $(\sim 8 f s)$ high-current $(0.4 \mathrm{kA})$ electron bunches with a normalized emittance of 8.5 and $6 \mathrm{~nm}$ in the two planes, respectively, and a brightness of $1.7 \times 10^{19} \mathrm{Arad}^{-2} \mathrm{~m}^{-2}$ can be obtained for realistic parameters [22].

\section{WORLDWIDE IONIZATION INJECTION DEMONSTRATION EXPERIMENTS}

In the previous sections, we introduced ionization based electron injection from analytical and simulation points of view. Almost at the same time of the theoretical progress, ionization injection has been experimentally demonstrated and optimized both in laser-driven and beam-driven wakefield acceleration. To our knowledge the first intentioned experimental studies of ionization injection in wakefield acceleration is by $\mathrm{Oz}$ et al. [23], where the wakefield is driven by an electron beam of $28.5 \mathrm{GeV}$ energy in a Lithium vapor column. Li plasma electrons act as the background electrons and support the wake. The doped Helium atoms are used as the ionization injection source since the higher ionization potential of He atoms makes them being ionized only inside the wakefield. As the wake amplitude is increased, the ionization induced Helium electron trapping is observed. Some electrons gain up to $7.6 \mathrm{GeV}$ energy in a $30.5 \mathrm{~cm}$ plasma. After that the ionization injections in laser-driven wakefield were also reported by three groups independently in 2010 [24-26].

Pak et al. [24] observed continuous ionization injection in their experiment above an initial laser intensity threshold (e.g. $a_{0}=2.35$, still less than the laser intensity threshold for self-injection) and electrons with maximum energy up to $110 \mathrm{MeV}$ were observed. The injection is due to the ionization of K-Shell of nitrogen in a He background plasma. Reducing the laser intensity to $a_{0}=1.64$ the low energy part of the spectrum disappeared and quasi-monoenergetic spectrum appeared due to the ionization stopping because of the lower laser intensity after some propagation. However, the spectrum was still very broad.

McGuffey et al. [25] used targets composed of helium and controlled amounts of various gases. In some of their experiments with laser power of 30TW and 120TW level, they found the addition of a higher Z additive (such as Ar, N2, Ne) can increase the beam charge by as much as an order of magnitude compared to pure helium at the same electron density and decrease the beam divergence from $5.1 \pm 1.0$ to $2.9 \pm 0.8 \mathrm{mrad}$. Their simulations for the experiments showed that electron beams with energy up to $150 \mathrm{MeV}$ were generated. The spectra were basically broad (more than $20 \%$ ).

By using higher laser power (60fs, 110TW) and longer acceleration length (1.3cm), Clayton et al. [26] accelerated electrons up to $1.45 \mathrm{GeV}$ in a regime of matched-beam, self-guided laser propagation and ionization-induced injection. They used $3 \%$ amounts of $\mathrm{CO}_{2}$ gas as injection gas. The background plasma density from He is $1.3 \times 10^{18} \mathrm{~cm}^{-3}$. At this low density and for $a_{0} \leq 4$, only electrons born inside the ion bubble are expected to be trapped via ionization-induced injection. Computer simulations confirmed that it is the K-shell electrons of oxygen that are ionized and injected into the wake and accelerated to beyond $1 \mathrm{GeV}$ energy. The spectrum again shows very broad character as shown in Fig. 8 (see beam k).

To reduce the energy spread of the electrons accelerated in LWFA by ionization injection, two groups (Liu [27] and Pol- 


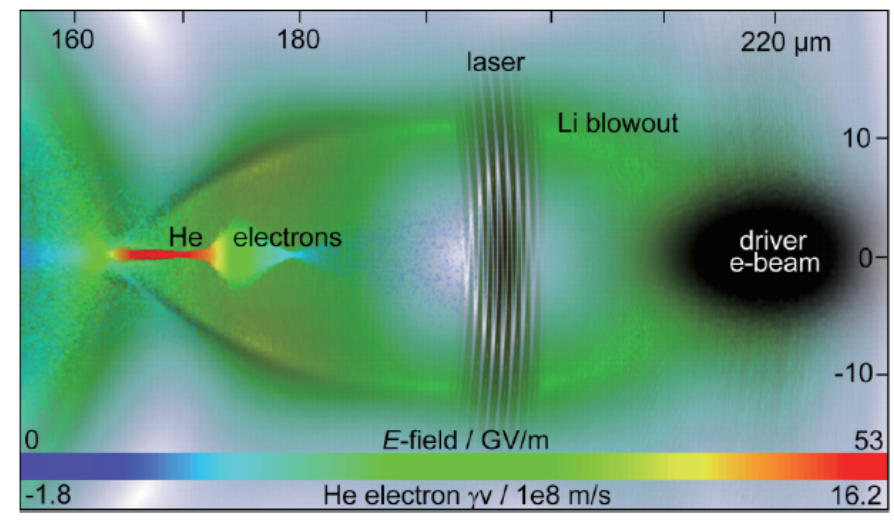

FIG. 9: Simulation results show how an electron driver ionizes Li gas and generates a Li blowout with an electron density of $n_{e}(L i)=$ $3.3 \times 10^{17} \mathrm{~cm}^{-3}$. The Ti:sapphire laser pulse with a duration of $8 \mathrm{fs}$ and $a_{0}=0.018$ is located at the end of the first half of the blowout at the electric fields turning point, and has already ionized some He electrons, which are then trapped and accelerated.

lock [28]) have used a similar scheme to separate ionization injection stage from acceleration stage and obtained quasimonoenergetic electron acceleration. In Liu's experiment [27], electrons with a Maxwellian spectrum, generated from the first LWFA assisted by ionization-induced injection, were seeded into the second LWFA with a 3-mm-thick gas cell. The first stage was 1-mm thick and filled with $6 \%$ oxygen gas and $94 \%$ helium gas flow, the second stage with variable thickness from 1 to $3 \mathrm{~mm}$ was filled with pure He gas. The $40 \sim 60 \mathrm{TW}$ laser pulses were used in the experiments. The plasma densities were $\sim 5.7 \times 10^{18} \mathrm{~cm}^{-3}$ in the first gass cell stage and $\sim 2.5 \times 10^{18} \mathrm{~cm}^{-3}$ in the second one, respectively. Finally a quasi-monoenergetic electron bunch peaked at an energy of $0.8 \mathrm{GeV}$ was observed with $25 \%$ energy spread, 2.6 mrad divergence, and $\sim 3.7 p C$ charge. In Pollock's scheme [28], the gas cell is comprised of a $3 \mathrm{~mm}$ injection stage, filled with a mixture of $99.5 \%$ helium and $0.5 \%$ nitrogen gas, separated by a $1 \mathrm{~mm}$ diameter aperture from an immediately adjacent $5 \mathrm{~mm}$ acceleration stage containing pure He. Plasma density of $3 \times 10^{18} \mathrm{~cm}^{-3}$ in each stage for a coupled laser power of $40 \mathrm{TW}$ were used. Finally a $460 \pm 25 \mathrm{MeV}$ electron beam containing $\sim 35 p C$ of charge was observed. PIC simulations for both these two experiments show that ionization induced injection is the main mechanism for electrons trapping in the wake in the first stage. The following acceleration stage is only used to boost the beam energy and reduce the relative energy spread. However, due to the large thickness of the first gas jet, electron beams injected by this two stage scheme are inevitably with large absolute energy spread as we mentioned before. Two-color ionization injection scheme may solve this issues. However, no experiments on this have been reported yet.

At last, we mention that recently in the laboratory for laser plasma at Shanghai Jiao Tong university, an experimental group has obtained quasi-monoenergetic electron beam with central energy of $1.14 \mathrm{GeV}$ energy spread of $7 \%$ from a single stage by using self-truncated ionization injection scheme which was discussed before in Section II B [29]. A preliminary result from the same group was recently published in Optics Express [13]. They use a mixed gas with $0.3 \%$ nitrogen and $99.7 \%$ helium gas. Upon the interaction of 30-TW, 30-fs laser pulses with a gas jet of the above gas mixture, $>300$ MeV quasi-monoenergetic electron beams were generated at a plasma densities of $3.3-8.5 \times 10^{18} \mathrm{~cm}^{-3}$.

\section{FURTHER DEVELOPMENT OF IONIZATION INDUCED ELECTRON INJECTION}

Due to the relatively simple experimental operation and flexible tunability, ionization injection is suggested as a very promising injection scheme in LWFA. Besides the above mentioned researches, it has also been modified and improved for other applications.

Hidding et al. recently has proposed a concept named plasma photocathode emission and used it in a beam-driven plasma blowout regime as shown in Fig. 9 [30, 31]. The scheme is similar as the one we mentioned before for low emittance beam generation by using two-color laser pulse. The difference is that in the current scheme the first driver laser pulse is replaced by a relativistic electron beam with charge of $300 \mathrm{pC}$ and energy of $200 \mathrm{MeV}$. Same as the first ionization injection demonstrated experiment, the background is a Lithium plasma with density of $3.3 \times 10^{17} \mathrm{~cm}^{-3}$ and the injection gas is Helium. So a very low laser intensity $(a=0.018)$ can be used to ionize He and make electron injection. In their simulation they found a beam with normalized emittance of $\varepsilon_{N} \simeq 4 \times 10^{-8} \mathrm{mrad}$ can be obtained, which opens up the possibility of its applications in future laser plasma accelerator based free electron $\mathrm{x}$-ray lasers (FEL).

Another two pulse ionization injection scheme was proposed by Bourgeois et al. [32] An important feature of their scheme is that the parameters of the injecting laser pulse are adjusted so that it diffracts faster than the driving laser pulse, meaning that the injection laser pulse intensity will remain high and hence electron trapping will occur in only a localized region. In contrast, 
the driving laser pulse can be guided in a plasma channel or a hollow capillary waveguide, allowing the trapped electrons to be accelerated to high energy. Particle-in-cell simulations show controlled injection and acceleration of electrons to an energy of $370 \mathrm{MeV}$, a relative energy spread of $2 \%$, and a normalized transverse emittance of $2.0 \mu \mathrm{m}$.

The final electron emittance not only depends on the initial injection process, but also on the following electrons' dynamics in phase space (mainly affected by Betatron motion). By using theory and PIC simulations, Xu et al. have recently studied phase-space dynamics of electrons injection by ionization mechanism in plasma-based accelerators [33]. They found that the injection process involves both longitudinal and transverse phase mixing, leading initially to a rapid growth of emittance and following oscillations, decay and a slow growth up to saturation. Electrons ionized at the same time can reside over a large range of $\psi$ of the wake, and thus feel a range of longitudinal wakefield $E_{x}$. The difference of $E_{x}$ cause the longitudinal phase mixing to the ionization injected electrons because of different Betatron frequency. Electrons ionized at different times $\left(t_{\text {ion }}\right)$ can also reside within the same longitudinal beam slice. Transverse mixing can occur within a slice due to the different phase-space distributions including different initial energies of the electrons after injection. These two phase mixing processes are responsible for the complex emittance dynamics. From theory and simulations they also found an optimal acceleration distance $\left(x_{0}=\left(E_{x} / \sigma_{E_{x}}\right) \sigma_{x}\right)$ to achieve minimum emittance for the trapped electrons, where $\sigma_{x}^{2}=<\left(s_{i}-<s_{i}>\right)^{2}>$ and $\sigma_{E_{x}}^{2}=<\left(E_{x}-<E_{x}>\right)^{2}>, s_{i}$ and $E_{x}$ represent the ionization position and the local wakefield intensity, respectively.

\section{CONCLUSION}

In this chapter, we have introduced a relatively simple electron injection method in laser wakefield acceleration, which relies on the ionization of the inner shell electrons of a High $\mathrm{Z}$ gas. These electrons are protected by the mother ions while passing through the deceleration phase of the wake. When they arrive at the appropriate phase of the wake they are ionized by the strong laser electric field there and then are trapped in the wake for following acceleration. Improved ionization schemes such as the two-color scheme and the transverse pulse ionization injection scheme are proposed by several groups to reduce the final energy spread and transverse emittance of the accelerated electron beams. Although such schemes can improve some aspects of the qualities of the beams, none of them can make simultaneous high quality for both longitudinal and transverse emittances. More theoretical and experimental investigations are necessary to make the LWFA beam appropriate for wide applications, such as new generation radiation source, TeV collider, and so on. Especially for external beam radiotherapy applications, the challenge is to produce a mount of high quality low energy electron beams. For this purpose, high repetition low power laser driven wakefield acceleration may be a better selection, in which ionization injection is also a good scheme since it can separate the high quality injection process from the acceleration process. At the same time, the high energy photon radio therapy such as $\mathrm{X}$ rays or $\gamma$ rays can also benefit by the ionization injection scheme. Electrons with high quality can radiate $\mathrm{X}$ rays through betatron oscillation [4], plasma channel guided oscillation [34] or Bremstrahlung radiation. For this purpose more work of improving the yield and quality of the radiations should be done.

\section{Acknowledgments}

This work was supported by the National Basic Research Program of China (Grant No. 2013CBA01504), the National Natural Science Foundation of China (Grant Nos. 11421064, 11374209, 11374210 and 11405107), the MOST international collaboration project (Grant No. 2014DFG02330). M.C. appreciates supports from National 1000 Youth Talent Project of China.

[1] E. Esarey, et al. Rev. Mod. Phys. 81, 1229 (2009).

[2] W.P. Leemans, and E. Esarey, Phys. Today 62, 44 (2009).

[3] S.M. Hooker, Nature Photon. 7, 775 (2013).

[4] S. Corde, et al. Rev. Mod. Phys. 85, 1 (2013).

[5] E. Esarey, et al. Phys. Rev. Lett. 79, 2682 (1997).

[6] J. Faure, et al. Nature 431, 541 (2004).

[7] E. Esarey, M. Pilloff, Phys. Plasmas 2, 1432 (1995).

[8] D. Umstadter, J.K. Kim, and E. Dodd, Phys. Rev. Lett. 76, 2073 (1996).

[9] M. Chen et al., J. Appl. Phys., 99, 056109 (2006).

[10] M. Chen et al., Phys. Plasmas 19, 033101 (2012).

[11] S. Kalmykov, S. A. Yi, V. Khudik, and G. Shvets, Phys. Rev. Lett. 103, 135004 (2009).

[12] M. Zeng et al., Phys. Plasmas 21, 030701 (2014).

[13] S. Li, et al., Optics Express, 22, 29578 (2014).

[14] C. Xia, et al., Phys. Plasmas 18, 113101 (2011). 
[15] M. Zeng, et al., Phys. Rev. Lett. 114, 084801 (2015).

[16] J.S. Qian, X.Z. Zhang, and M.L. Tian, Journal of Xi' an Jiaotong University (Medical Sciences) 1, 122 (2013).

[17] T. Shouman, Z. El-Taher, Journal of the Egypitan Nat. Cancer Inst., 6, 202 (2004).

[18] L.-L. Yu et al., Phys. Rev. Lett. 112, 125001 (2014).

[19] X.L. Xu, et al., Phys. Rev. ST Accel. Beams 17, 061301 (2014).

[20] M. Chen et al., AIP Conf. Proc. 1507, 262 (2012).

[21] M. Chen et al., Phys. Rev. ST Accel. Beams 17, 051303 (2014).

[22] F. Li, et al., Phys. Rev. Lett. 111, 015003 (2013).

[23] E. Oz, S. Deng, T. Katsouleas, et al., Phys. Rev. Lett. 98, 084801 (2007).

[24] A. Pak, et al., Phys. Rev. Lett. 104, 025003 (2010).

[25] B.C. McGuffey, et al., Phys. Rev. Lett. 104, 025004 (2010).

[26] C.E. Clayton, et al., Phys. Rev. Lett. 105, 105003 (2010).

[27] J.S. Liu, et al., Phys. Rev. Lett. 107, 035001 (2011).

[28] B. B. Pollock, et al., Phys. Rev. Lett. 107, 045001 (2011).

[29] M. Mirzaie, S. Li, M. Zeng, et al., Sci. Rep. 5, 14659; doi:10.1038/srep14659 (2015).

[30] B. Hidding, et al., Phys. Rev. Lett. 108, 035001 (2012).

[31] Y. Xi, et al., Phys. Rev. ST Accel. Beams 16, 031303 (2013).

[32] N. Bourgeois, et al., Phys. Rev. Lett. 111, 155004 (2013).

[33] X.L. Xu, et al., Phys. Rev. Lett. 112, 035003 (2014).

[34] M. Chen, J. Luo, F.Y. Li, et al., Light: Science and Applications, accepted article preview 28 August 2015; e16015; doi: 10.1038/1sa.2016.15. 